\title{
Existence of groundstates for Choquard type equations with Hardy-Littlewood-Sobolev critical exponent
}

Xiaowei $\mathrm{Li}^{1}$ and Feizhi Wang ${ }^{1 *}$

\section{"Correspondence:}

wangfz@ytu.edu.cn

${ }^{1}$ School of Mathematics and Information Sciences, Yantai University, Yantai 264005,

Shandong, P.R. China

\begin{abstract}
In this paper, we consider a class of Choquard equations with Hardy-Littlewood-Sobolev lower or upper critical exponent in the whole space $\mathbb{R}^{N}$. We combine an argument of L. Jeanjean and H. Tanaka (see (Proc. Am. Math. Soc. 131:2399-2408, 2003) with a concentration-compactness argument, and then we obtain the existence of ground state solutions, which extends and complements the earlier results.
\end{abstract}

Keywords: Choquard equation; Nonlocal critical growth; Pohozăev-Palais-Smale sequence; Hardy-Littlewood-Sobolev inequality

\section{Introduction}

In this paper, we consider the following nonlinear Choquard problem:

$$
-\Delta u+u=\left(I_{\alpha} * F(u)\right) f(u), \quad u \in H^{1}\left(\mathbb{R}^{N}\right),
$$

where $N \geq 3,0<\alpha<N, I_{\alpha}$ is a Riesz potential

$$
I_{\alpha}(x)=\frac{\Gamma\left(\frac{N-\alpha}{2}\right)}{\Gamma\left(\frac{\alpha}{2}\right) \pi^{\frac{N}{2}} 2^{\alpha}|x|^{N-\alpha}}:=\frac{\hat{C}}{|x|^{N-\alpha}}
$$

with $\Gamma(s)=\int_{0}^{+\infty} x^{s-1} e^{-x} d x, s>0, F \in C^{1}(\mathbb{R}, \mathbb{R})$, and $f:=F^{\prime}$. Problem (P) can be studied by the variational method. It is the Euler-Lagrange equation of the functional

$$
\Phi(u)=\frac{1}{2} \int_{\mathbb{R}^{N}}\left[|\nabla u|^{2}+u^{2}\right] d x-\frac{1}{2} \int_{\mathbb{R}^{N}}\left(I_{\alpha} * F(u)\right) F(u) d x .
$$

As we know, a large number of works have been devoted to the problem like (P). We refer the readers to $[2,4,5,7-10,12-15]$ and the references therein.

Especially, in [8], under the following conditions:

$\left(\mathrm{MS}_{1}\right) f \in C(\mathbb{R}, \mathbb{R})$ and there exists $C>0$ such that, for every $s \in \mathbb{R}$,

$$
|s f(s)| \leq C\left(|s|^{\frac{N+\alpha}{N}}+|s|^{\frac{N+\alpha}{N-2}}\right) ;
$$

(c) The Author(s) 2021. This article is licensed under a Creative Commons Attribution 4.0 International License, which permits use, sharing, adaptation, distribution and reproduction in any medium or format, as long as you give appropriate credit to the original author(s) and the source, provide a link to the Creative Commons licence, and indicate if changes were made. The images or other third party material in this article are included in the article's Creative Commons licence, unless indicated otherwise in a credit line to the material. If material is not included in the article's Creative Commons licence and your intended use is not permitted by statutory regulation or exceeds the permitted use, you will need to obtain permission directly from the copyright holder. To view a copy of this licence, visit http://creativecommons.org/licenses/by/4.0/. 
$\left(\mathrm{MS}_{2}\right)$

$$
\lim _{s \rightarrow 0} \frac{F(s)}{|s|^{\frac{N+\alpha}{N}}}=0 \quad \text { and } \quad \lim _{s \rightarrow \infty} \frac{F(s)}{|s|^{\frac{N+\alpha}{N-2}}}=0
$$

$\left(\mathrm{MS}_{3}\right)$ There exists $s_{0} \in \mathbb{R} \backslash\{0\}$ such that $F\left(s_{0}\right) \neq 0$,

Moroz and Schaftingen proved the existence of a ground state solution. They employed a method introduced by L. Jeanjean, where a key step is to construct Palais-Smale sequences that satisfy asymptotically the Pohozǎev identity [3]. Note that assumption $\left(\mathrm{MS}_{2}\right)$ is called subcritical. The constant $\frac{N+\alpha}{N}$ is termed the lower-critical exponent and $\frac{N+\alpha}{N-2}$ is termed the upper-critical exponent in the sense of Hardy-Littlewood-Sobolev inequality. In [14], Seok considered that problem $(\mathrm{P})$ with $F(u)$ is doubly critical, i.e.,

$$
F(u)=\frac{1}{p}|u|^{p}+\frac{1}{q}|u|^{q},
$$

where $p=\frac{N+\alpha}{N}$ and $q=\frac{N+\alpha}{N-2}$. In this situation,

$$
\lim _{s \rightarrow 0} \frac{F(s)}{|s|^{\frac{N+\alpha}{N}}}=\frac{1}{p} \neq 0 \quad \text { and } \quad \lim _{s \rightarrow \infty} \frac{F(s)}{|s|^{\frac{N+\alpha}{N-2}}}=\frac{1}{q} \neq 0 .
$$

He showed the existence of nontrivial solutions of the nonlinear Choquard equation if $\alpha+4<N$.

In the following we give our main result.

Theorem 1.1 Suppose that $N \geq 3, p, q \in\left[\frac{N+\alpha}{N}, \frac{N+\alpha}{N-2}\right]$ and

$$
F(u)=\frac{1}{p}|u|^{p}+\frac{1}{q}|u|^{q} .
$$

Then problem $(\mathrm{P})$ has at least a ground state solution $u \in H^{1}\left(\mathbb{R}^{N}\right)$ provided one of the following conditions holds:

(1) $q=\frac{N+\alpha}{N-2}, N \geq 4$, and $p \in\left(\frac{N+\alpha}{N}, \frac{N+\alpha}{N-2}\right)$ or $N=3$ and $p \in\left(1+\frac{\alpha}{N-2}, \frac{N+\alpha}{N-2}\right)$;

(2) $p=\frac{N+\alpha}{N}, N>4+\alpha$, and $q \in\left(\frac{N+\alpha}{N}, \frac{N+\alpha}{N-2}\right]$ or $N<4+\alpha$ and $q \in\left(\frac{N+\alpha}{N}, \frac{N+\alpha+4}{N}\right)$.

Remark 1.1 By conditions (1) and (2), it is easy to see that

$$
\lim _{s \rightarrow 0} \frac{F(s)}{|s|^{\frac{N+\alpha}{N}}}=0 \quad \text { and } \quad \lim _{s \rightarrow \infty} \frac{F(s)}{|s|^{N-2}}=\frac{1}{q} \neq 0
$$

and

$$
\lim _{s \rightarrow 0} \frac{F(s)}{|s|^{\frac{N+\alpha}{N}}}=\frac{1}{p} \neq 0 \quad \text { and } \quad \lim _{s \rightarrow \infty} \frac{F(s)}{|s|^{N-2}}=0 \text { or } \frac{1}{q},
$$

respectively.

We denote the strong and the weak convergence in $H^{1}\left(\mathbb{R}^{N}\right)$ by $\rightarrow$ and $\rightarrow$, respectively. Set $\|u\|:=\left[\int_{\mathbb{R}^{N}}\left(|\nabla u|^{2}+u^{2}\right) d x\right]^{1 / 2}$ and $|u|_{q}:=\left[\int_{\mathbb{R}^{N}}|u|^{q} d x\right]^{1 / q}$ for $1<q<\infty$. As for the 
Choquard equation, the Hardy-Littlewood-Sobolev inequality (see [6] and [7]) implies that the nonlocal term is well defined for $u \in H^{1}\left(\mathbb{R}^{N}\right)$ and $\Phi$ is continuously differentiable on $H^{1}\left(\mathbb{R}^{N}\right)$. Clearly, $u=0$ is a trivial solution of $(\mathrm{P})$. The solutions of $(\mathrm{P})$ must verify the Pohozǎev identity, as was proved in [8, Corollary 3.5]. In our case, the Pohozǎev identity reads as follows:

$$
\mathcal{P}(u):=\frac{N-2}{2} \int_{\mathbb{R}^{N}}|\nabla u|^{2} d x+\frac{N}{2} \int_{\mathbb{R}^{N}} u^{2} d x-\frac{N+\alpha}{2} \int_{\mathbb{R}^{N}}\left(I_{\alpha} * F(u)\right) F(u) d x=0 .
$$

We call any weak solution $u \in H^{1}\left(\mathbb{R}^{N}\right) \backslash\{0\}$ of $(\mathrm{P})$ a groundstate of $(\mathrm{P})$ if

$$
\Phi(u)=c_{0}:=\inf \left\{\Phi(v): v \in H^{1}\left(\mathbb{R}^{N}\right) \backslash\{0\} \text { is a solution of }(\mathrm{P})\right\} .
$$

Because problem $(\mathrm{P})$ contains nonlocal critical nonlinearities in $\mathbb{R}^{N}$, there are more difficulties to overcome. One difficulty is the embedding of $H^{1}\left(\mathbb{R}^{N}\right)$ into $L^{q}\left(\mathbb{R}^{N}\right)$ which is not compact, where $2 \leq q \leq 2^{*}$. As a consequence, the corresponding functional of (P) does not satisfy the Palais-Smale condition; we overcome the lack of compactness by studying the problem in $H_{r}^{1}\left(\mathbb{R}^{N}\right)$ :

$$
H_{r}^{1}\left(\mathbb{R}^{N}\right)=\left\{u \in H^{1}\left(\mathbb{R}^{N}\right): u(x)=u(|x|)\right\}
$$

which embeds compactly into $L^{q}\left(\mathbb{R}^{N}\right)$. By standard arguments (the principle of symmetric criticality; see [11] or [16, Theorem 1.28]), one has that a critical point $u \in H_{r}^{1}\left(\mathbb{R}^{N}\right)$ for the functional $\Phi(u)$ of $(\mathrm{P})$ is also a critical point in $H^{1}\left(\mathbb{R}^{N}\right)$. We say that $\left\{u_{n}\right\} \subset H^{1}\left(\mathbb{R}^{N}\right)$ is a Pohozǎev-Palais-Smale sequence for $\Phi \in C^{1}\left(H^{1}\left(\mathbb{R}^{N}\right), \mathbb{R}\right)$ at level $c \in \mathbb{R}$ if and only if $\left\{u_{n}\right\}$ satisfies $\Phi\left(u_{n}\right) \rightarrow c, \Phi^{\prime}\left(u_{n}\right) \rightarrow 0$, and $\mathcal{P}\left(u_{n}\right) \rightarrow 0$ as $n \rightarrow \infty$. Following the strategy in [3], we obtain that there exists a Pohozarev-Palais-Smale sequence for $\Phi$, with $c$ confined in a suitable range. To ensure that the mini-max levels stay in a certain range, we make some careful computation in Sect. 2, which is crucial in our approach. Then, we make full use of three limit formulas in the Pohozarev-Palais-Smale sequence and prove that this sequence has a strongly convergent subsequence.

\section{Preliminaries}

In the following, we recall the well-known Hardy-Littlewood-Sobolev inequality (see in [6, Theorem 4.3]).

Proposition 2.1 (Hardy-Littlewood-Sobolev inequality) Let $r, s>1$ and $\alpha \in(0, N)$ with $\frac{1}{r}+\frac{1}{s}=1+\frac{\alpha}{N}$. Then there exists $C>0$ depending only on $N, \alpha, r$ such that, for any $f \in L^{r}\left(\mathbb{R}^{N}\right)$ and $g \in L^{s}\left(\mathbb{R}^{N}\right)$,

$$
\left|\int_{\mathbb{R}^{N}} \int_{\mathbb{R}^{N}} \frac{f(x) g(y)}{|x-y|^{N-\alpha}} d x d y\right| \leq C(N, \alpha, r)\|f\|_{L^{r}\left(\mathbb{R}^{N}\right)}\|g\|_{L^{s}\left(\mathbb{R}^{N}\right)} .
$$

Lemma 2.1 Suppose that $N \geq 3$ and $p, q \in\left[\frac{N+\alpha}{N}, \frac{N+\alpha}{N-2}\right]$. Let $\left\{v_{n}\right\} \subset H_{r}^{1}\left(\mathbb{R}^{N}\right)$ be a sequence converging weakly to 0 as $n \rightarrow \infty$. If $\frac{2(N+\alpha)}{N}<p+q<\frac{2(N+\alpha)}{N-2}$, then

$$
\int_{\mathbb{R}^{N}}\left(I_{\alpha} *\left|v_{n}\right|^{p}\right)\left|v_{n}\right|^{q} d x \rightarrow 0, \quad \text { as } n \rightarrow \infty .
$$


Proof Since $v_{n} \rightarrow 0$ in $H_{r}^{1}\left(\mathbb{R}^{N}\right)$ and $2<\frac{N(p+q)}{N+\alpha}<\frac{2 N}{N-2}$, we have

$$
v_{n} \rightarrow 0 \quad \text { in } L^{\frac{N(p+q)}{N+\alpha}}\left(\mathbb{R}^{N}\right)
$$

see [16, Corollary 1.25]. By the Hardy-Littlewood-Sobolev inequality with $r=\frac{p+q}{p} \frac{N}{N+\alpha}$ and $t=\frac{p+q}{q} \frac{N}{N+\alpha}$, we obtain

$$
\begin{aligned}
\left.\left|\int_{\mathbb{R}^{N}}\left(I_{\alpha} *\left|v_{n}\right|^{p}\right)\right| v_{n}\right|^{q} d x \mid & \leq C\left\|\left|v_{n}\right|^{p}\right\|_{L^{r}\left(\mathbb{R}^{N}\right)}\left\|\left|v_{n}\right|^{q}\right\|_{L^{t}\left(\mathbb{R}^{N}\right)} \\
& =C\left(\int_{\mathbb{R}^{N}}\left|v_{n}\right|^{\frac{N(p+q)}{N+\alpha}} d x\right)^{\frac{N+\alpha}{N}} \rightarrow 0,
\end{aligned}
$$

where $C$ is a positive constant. The proof is finished.

Remark $2.1 p, q$ that appear in Theorem 1.1 satisfy

$$
\frac{2(N+\alpha)}{N}<p+q<\frac{2(N+\alpha)}{N-2} .
$$

The constant $\mathcal{S}_{1}$ is defined by

$$
\mathcal{S}_{1}:=\inf \left\{\frac{|\nabla u|_{2}^{2}}{\left[\int_{\mathbb{R}^{N}}\left(I_{\alpha} *|u|^{q}\right)|u|^{q} d x\right]^{1 / q}}: u \in H^{1}\left(\mathbb{R}^{N}\right) \backslash\{0\}\right\}
$$

and is attained by the functions

$$
U_{\varepsilon}(x)=\frac{C \varepsilon^{(N-2) / 2}}{\left(\varepsilon^{2}+|x|^{2}\right)^{(N-2) / 2}}
$$

where $\varepsilon>0$ (see in [6]). We define a cutoff function $\varphi(x)$ by

$$
\varphi(x)= \begin{cases}1, & x \in B_{1} \\ 2-|x|, & x \in B_{2} \backslash B_{1} \\ 0, & x \in \mathbb{R}^{N} \backslash B_{2}\end{cases}
$$

where $B_{1}=\left\{x \in \mathbb{R}^{N}:|x| \leq 1\right\}$ and $B_{2}=\left\{x \in \mathbb{R}^{N}:|x| \leq 2\right\}$. Set

$$
u_{\varepsilon}=u_{\varepsilon}(x)=\varphi(x) \cdot U_{\varepsilon}(x) .
$$

Then we have the following lemma.

Lemma 2.2 Suppose that $N \geq 3$ and $p, q \in\left[\frac{N+\alpha}{N}, \frac{N+\alpha}{N-2}\right]$. Then there exists a positive constant $\varepsilon_{0}$ such that if $\varepsilon \in\left(0, \varepsilon_{0}\right)$ then

$$
\Phi\left(t u_{\varepsilon}\right)<\frac{1}{2}\left(1-\frac{1}{q}\right) q^{\frac{1}{q-1}} \mathcal{S}_{1}^{\frac{N+\alpha}{2+\alpha}} \quad \text { for all } t \geq 0
$$

provided $q=\frac{N+\alpha}{N-2}$ and one of the following conditions holds: 
Li and Wang Boundary Value Problems

(2021) 2021:102

Page 5 of 20

(1) $N \geq 4$ and $p \in\left[\frac{N+\alpha}{N}, \frac{N+\alpha}{N-2}\right)$;

(2) $N=3$ and $p \in\left(1+\frac{\alpha}{N-2}, \frac{N+\alpha}{N-2}\right)$.

Proof According to the definition of $\Phi$ and $u_{\varepsilon}$, we have

$$
\begin{aligned}
\Phi\left(t u_{\varepsilon}\right)= & \frac{t^{2}}{2} \int_{\mathbb{R}^{N}}\left[\left|\nabla u_{\varepsilon}\right|^{2}+u_{\varepsilon}^{2}\right] d x-\frac{1}{2} \int_{\mathbb{R}^{N}}\left(I_{\alpha} * F\left(t u_{\varepsilon}\right)\right) F\left(t u_{\varepsilon}\right) d x \\
= & \frac{t^{2}}{2} \int_{\mathbb{R}^{N}}\left|\nabla u_{\varepsilon}\right|^{2} d x+\frac{t^{2}}{2} \int_{\mathbb{R}^{N}} u_{\varepsilon}^{2} d x-\frac{t^{2 p}}{2 p^{2}} \int_{\mathbb{R}^{N}}\left(I_{\alpha} *\left|u_{\varepsilon}\right|^{p}\right)\left|u_{\varepsilon}\right|^{p} d x \\
& -\frac{t^{p+q}}{p q} \int_{\mathbb{R}^{N}}\left(I_{\alpha} *\left|u_{\varepsilon}\right|^{p}\right)\left|u_{\varepsilon}\right|^{q} d x-\frac{t^{2 q}}{2 q^{2}} \int_{\mathbb{R}^{N}}\left(I_{\alpha} *\left|u_{\varepsilon}\right|^{q}\right)\left|u_{\varepsilon}\right|^{q} d x \\
= & \frac{t^{2}}{2} \int_{\mathbb{R}^{N}}\left|\nabla u_{\varepsilon}\right|^{2} d x+\frac{t^{2}}{2} \int_{\mathbb{R}^{N}} u_{\varepsilon}^{2} d x-\frac{t^{2 p}}{2 p^{2}} \mathcal{L}-\frac{t^{p+q}}{p q} \mathcal{H}-\frac{t^{2 q}}{2 q^{2}} \mathcal{M},
\end{aligned}
$$

where

$$
\begin{aligned}
& \mathcal{L}:=\int_{\mathbb{R}^{N}} \int_{\mathbb{R}^{N}} \frac{\hat{C}\left|u_{\varepsilon}(x)\right|^{p}\left|u_{\varepsilon}(y)\right|^{p}}{|x-y|^{N-\alpha}} d x d y, \\
& \mathcal{H}:=\int_{\mathbb{R}^{N}} \int_{\mathbb{R}^{N}} \frac{\hat{C}\left|u_{\varepsilon}(x)\right|^{p}\left|u_{\varepsilon}(y)\right|^{q}}{|x-y|^{N-\alpha}} d x d y
\end{aligned}
$$

and

$$
\mathcal{M}:=\int_{\mathbb{R}^{N}} \int_{\mathbb{R}^{N}} \frac{\hat{C}\left|u_{\varepsilon}(x)\right|^{q}\left|u_{\varepsilon}(y)\right|^{q}}{|x-y|^{N-\alpha}} d x d y
$$

By [1] (see also [16]), the following asymptotic estimates hold as $\varepsilon$ is small enough:

$$
\int_{\mathbb{R}^{N}}\left|\nabla u_{\varepsilon}\right|^{2} d x=\mathcal{S}_{1}^{\frac{N+\alpha}{2+\alpha}}+O\left(\varepsilon^{N-2}\right)
$$

and

$$
h(\varepsilon):=\int_{\mathbb{R}^{N}}\left|u_{\varepsilon}\right|^{2} d x= \begin{cases}c \varepsilon^{2}+O\left(\varepsilon^{N-2}\right), & \text { if } N \geq 5 \\ c \varepsilon^{2}|\log \varepsilon|+O\left(\varepsilon^{2}\right), & \text { if } N=4 \\ c \varepsilon+O\left(\varepsilon^{2}\right), & \text { if } N=3\end{cases}
$$

where $c$ is a positive constant.

In the following we estimate the convolution terms $\mathcal{L}, \mathcal{H}$, and $\mathcal{M}$, respectively.

Case $\mathcal{L}$ :

$$
\begin{aligned}
\mathcal{L} & =\int_{\mathbb{R}^{N}} \int_{\mathbb{R}^{N}} \frac{\hat{C}\left|u_{\varepsilon}(x)\right|^{p}\left|u_{\varepsilon}(y)\right|^{p}}{|x-y|^{N-\alpha}} d x d y \\
& =\int_{B_{2}} \int_{B_{2}} \frac{\hat{C}\left|u_{\varepsilon}(x)\right|^{p}\left|u_{\varepsilon}(y)\right|^{p}}{|x-y|^{N-\alpha}} d x d y \\
& \geq \int_{B_{1}} \int_{B_{1}} \frac{\hat{C}\left|u_{\varepsilon}(x)\right|^{p}\left|u_{\varepsilon}(y)\right|^{p}}{|x-y|^{N-\alpha}} d x d y
\end{aligned}
$$




$$
\begin{aligned}
= & \int_{B_{1}} \int_{B_{1}} \frac{\hat{C}\left|U_{\varepsilon}(x)\right|^{p}\left|U_{\varepsilon}(y)\right|^{p}}{|x-y|^{N-\alpha}} d x d y \\
= & \int_{B_{2}} \int_{B_{2}} \frac{\hat{C}\left|U_{\varepsilon}(x)\right|^{p}\left|U_{\varepsilon}(y)\right|^{p}}{|x-y|^{N-\alpha}} d x d y-\int_{B_{2} \backslash B_{1}} \int_{B_{1}} \frac{\hat{C}\left|U_{\varepsilon}(x)\right|^{p}\left|U_{\varepsilon}(y)\right|^{p}}{|x-y|^{N-\alpha}} d x d y \\
& -\int_{B_{2} \backslash B_{1}} \int_{B_{2} \backslash B_{1}} \frac{\hat{C}\left|U_{\varepsilon}(x)\right|^{p}\left|U_{\varepsilon}(y)\right|^{p}}{|x-y|^{N-\alpha}} d x d y \\
= & \mathcal{L}_{1}-2 \mathcal{L}_{2}-\mathcal{L}_{3},
\end{aligned}
$$

where

$$
\begin{aligned}
& \mathcal{L}_{1}:=\int_{B_{2}} \int_{B_{2}} \frac{\hat{C}\left|U_{\varepsilon}(x)\right|^{p}\left|U_{\varepsilon}(y)\right|^{p}}{|x-y|^{N-\alpha}} d x d y, \\
& \mathcal{L}_{2}:=\int_{B_{2} \backslash B_{1}} \int_{B_{1}} \frac{\hat{C}\left|U_{\varepsilon}(x)\right|^{p}\left|U_{\varepsilon}(y)\right|^{p}}{|x-y|^{N-\alpha}} d x d y,
\end{aligned}
$$

and

$$
\mathcal{L}_{3}:=\int_{B_{2} \backslash B_{1}} \int_{B_{2} \backslash B_{1}} \frac{\hat{C}\left|U_{\varepsilon}(x)\right|^{p}\left|U_{\varepsilon}(y)\right|^{p}}{|x-y|^{N-\alpha}} d x d y
$$

By direct computation, we have, for $\varepsilon<1$,

$$
\begin{aligned}
\mathcal{L}_{1} & =O\left(\varepsilon^{-(N-2) p}\right) \int_{B_{2}} \int_{B_{2}} \frac{1}{\left(1+\left|\frac{x}{\varepsilon}\right|^{2}\right)^{\frac{(N-2) p}{2}}|x-y|^{N-\alpha}\left(1+\left|\frac{y}{\varepsilon}\right|^{2}\right)^{\frac{(N-2) p}{2}}} d x d y \\
& =O\left(\varepsilon^{N+\alpha-(N-2) p}\right) \int_{B_{2 / \varepsilon}} \int_{B_{2 / \varepsilon}} \frac{1}{\left(1+|x|^{2}\right)^{\frac{(N-2) p}{2}}|x-y|^{N-\alpha}\left(1+|y|^{2}\right)^{\frac{(N-2) p}{2}}} d x d y \\
& \geq O\left(\varepsilon^{N+\alpha-(N-2) p}\right) \int_{B_{\frac{1}{2}}}\left[\int_{B_{x, \frac{1}{2}}} \frac{1}{\left(1+|y|^{2}\right)^{\frac{(N-2) p}{2}}|x-y|^{N-\alpha}} d y\right] \frac{1}{\left(1+|x|^{2}\right)^{\frac{(N-2) p}{2}}} d x \\
& \geq O\left(\varepsilon^{N+\alpha-(N-2) p}\right) \int_{B_{\frac{1}{2}}}\left[\int_{B_{x, \frac{1}{2}}} \frac{1}{|x-y|^{N-\alpha}} d y\right] \frac{1}{\left(1+|x|^{2}\right)^{\frac{(N-2) p}{2}}} d x \\
& =O\left(\varepsilon^{N+\alpha-(N-2) p}\right) \int_{B_{\frac{1}{2}}} \frac{1}{|y|^{N-\alpha}} d y \cdot \int_{B_{\frac{1}{2}}} \frac{1}{\left(1+|x|^{2}\right)^{\frac{(N-2) p}{2}}} d x=O\left(\varepsilon^{N+\alpha-(N-2) p}\right) .
\end{aligned}
$$

We also have

$$
\mathcal{L}_{2}=O\left(\varepsilon^{(N-2) p}\right) \int_{B_{1}} \int_{B_{2} \backslash B_{1}} \frac{1}{\left(\varepsilon^{2}+|x|^{2}\right)^{\frac{(N-2) p}{2}}|x-y|^{N-\alpha}\left(\varepsilon^{2}+|y|^{2}\right)^{\frac{(N-2) p}{2}}} d x d y .
$$

By the Hardy-Littlewood-Sobolev inequality with $\frac{1}{r_{1}}+\frac{1}{s_{1}}=1+\frac{\alpha}{N}$ (see Proposition 2.1) and

$$
s_{1} \in\left(\frac{N}{(N-2) p}, \frac{2 N}{N+\alpha-(N-2) p}\right),
$$


Li and Wang Boundary Value Problems

(2021) 2021:102

Page 7 of 20

we have

$$
\begin{aligned}
\mathcal{L}_{2} \leq & O\left(\varepsilon^{(N-2) p}\right)\left(\int_{B_{2} \backslash B_{1}} \frac{1}{\left(\varepsilon^{2}+|x|^{2}\right)^{\frac{(N-2) p r_{1}}{2}}} d x\right)^{\frac{1}{r_{1}}} \\
& \times\left(\int_{B_{1}} \frac{1}{\left.\left(\varepsilon^{2}+|y|^{2}\right)^{\frac{(N-2) p s_{1}}{2}} d y\right)^{\frac{1}{s_{1}}}}\right. \\
\leq & O\left(\varepsilon^{\frac{N}{s_{1}}}\right)\left(\int_{0}^{\frac{1}{\varepsilon}} \frac{z^{N-1}}{\left(1+|z|^{2}\right)^{\frac{(N-2) p s_{1}}{2}}} d z\right)^{\frac{1}{s_{1}}} \\
\leq & O\left(\varepsilon^{\frac{N}{s_{1}}}\right)\left(1+\int_{1}^{\frac{1}{\varepsilon}}|z|^{N-1-(N-2) p s_{1}} d z\right)^{\frac{1}{s_{1}}}=O\left(\varepsilon^{\frac{N}{s_{1}}}\right) .
\end{aligned}
$$

We also get

$$
\begin{aligned}
\mathcal{L}_{3} & =O\left(\varepsilon^{(N-2) p}\right) \int_{B_{2} \backslash B_{1}} \int_{B_{2} \backslash B_{1}} \frac{1}{\left(\varepsilon^{2}+|x|^{2}\right)^{\frac{(N-2) p}{2}}|x-y|^{N-\alpha}\left(\varepsilon^{2}+|y|^{2}\right)^{\frac{(N-2) p}{2}}} d x d y \\
& \leq O\left(\varepsilon^{(N-2) p}\right) \int_{B_{2} \backslash B_{1}} \int_{B_{2} \backslash B_{1}} \frac{1}{|x|^{(N-2) p}|x-y|^{N-\alpha}|y|^{(N-2) p}} d x d y \\
& \leq O\left(\varepsilon^{(N-2) p}\right)\left[\int_{B_{2} \backslash B_{1}}|x|^{-\frac{2 N(N-2) p}{N+\alpha}} d x\right]^{\frac{N+\alpha}{N}}=O\left(\varepsilon^{(N-2) p}\right) .
\end{aligned}
$$

Combining (4), (6), and (7), we obtain

$$
\mathcal{L} \geq O\left(\varepsilon^{N+\alpha-(N-2) p}\right)-O\left(\varepsilon^{\frac{N}{s_{1}}}\right)-O\left(\varepsilon^{(N-2) p}\right) .
$$

Noting that $s_{1}>\frac{N}{(N-2) p}$,

$$
\mathcal{L} \geq O\left(\varepsilon^{N+\alpha-(N-2) p}\right)-O\left(\varepsilon^{\frac{N}{s_{1}}}\right) .
$$

Case $\mathcal{H}$ : It is easy to see that

$$
\begin{aligned}
\mathcal{H}= & \int_{\mathbb{R}^{N}} \int_{\mathbb{R}^{N}} \frac{\hat{C}\left|u_{\varepsilon}(x)\right|^{p}\left|u_{\varepsilon}(y)\right|^{q}}{|x-y|^{N-\alpha}} d x d y=\int_{B_{2}} \int_{B_{2}} \frac{\hat{C}\left|u_{\varepsilon}(x)\right|^{p}\left|u_{\varepsilon}(y)\right|^{q}}{|x-y|^{N-\alpha}} d x d y \\
\geq & \int_{B_{1}} \int_{B_{1}} \frac{\hat{C}\left|u_{\varepsilon}(x)\right|^{p}\left|u_{\varepsilon}(y)\right|^{q}}{|x-y|^{N-\alpha}} d x d y=\int_{B_{1}} \int_{B_{1}} \frac{\hat{C}\left|U_{\varepsilon}(x)\right|^{p}\left|U_{\varepsilon}(y)\right|^{q}}{|x-y|^{N-\alpha}} d x d y \\
= & \int_{B_{2}} \int_{B_{2}} \frac{\hat{C}\left|U_{\varepsilon}(x)\right|^{p}\left|U_{\varepsilon}(y)\right|^{q}}{|x-y|^{N-\alpha}} d x d y-\int_{B_{2} \backslash B_{1}} \int_{B_{1}} \frac{\hat{C}\left|U_{\varepsilon}(x)\right|^{p}\left|U_{\varepsilon}(y)\right|^{q}}{|x-y|^{N-\alpha}} d x d y \\
& -\int_{B_{1}} \int_{B_{2} \backslash B_{1}} \frac{\hat{C}\left|U_{\varepsilon}(x)\right|^{p}\left|U_{\varepsilon}(y)\right|^{q}}{|x-y|^{N-\alpha}} d x d y-\int_{B_{2} \backslash B_{1}} \int_{B_{2} \backslash B_{1}} \frac{\hat{C}\left|U_{\varepsilon}(x)\right|^{p}\left|U_{\varepsilon}(y)\right|^{q}}{|x-y|^{N-\alpha}} d x d y \\
= & \mathcal{H}_{1}-\mathcal{H}_{2}-\mathcal{H}_{3}-\mathcal{H}_{4},
\end{aligned}
$$


where

$$
\begin{aligned}
& \mathcal{H}_{1}:=\int_{B_{2}} \int_{B_{2}} \frac{\hat{C}\left|U_{\varepsilon}(x)\right|^{p}\left|U_{\varepsilon}(y)\right|^{q}}{|x-y|^{N-\alpha}} d x d y, \\
& \mathcal{H}_{2}:=\int_{B_{2} \backslash B_{1}} \int_{B_{1}} \frac{\hat{C}\left|U_{\varepsilon}(x)\right|^{p}\left|U_{\varepsilon}(y)\right|^{q}}{|x-y|^{N-\alpha}} d x d y, \\
& \mathcal{H}_{3}:=\int_{B_{1}} \int_{B_{2} \backslash B_{1}} \frac{\hat{C}\left|U_{\varepsilon}(x)\right|^{p}\left|U_{\varepsilon}(y)\right|^{q}}{|x-y|^{N-\alpha}} d x d y, \\
& \mathcal{H}_{4}:=\int_{B_{2} \backslash B_{1}} \int_{B_{2} \backslash B_{1}} \frac{\hat{C}\left|U_{\varepsilon}(x)\right|^{p}\left|U_{\varepsilon}(y)\right|^{q}}{|x-y|^{N-\alpha}} d x d y .
\end{aligned}
$$

For $\varepsilon<1$, we have

$$
\begin{aligned}
\mathcal{H}_{1} & =O\left(\varepsilon^{-\frac{(N-2)(p+q)}{2}}\right) \int_{B_{2}} \int_{B_{2}} \frac{1}{\left(1+\left|\frac{x}{\varepsilon}\right|^{2}\right)^{\frac{(N-2) p}{2}}|x-y|^{N-\alpha}\left(1+\left|\frac{y}{\varepsilon}\right|^{2}\right)^{\frac{(N-2) q}{2}}} d x d y \\
& =O\left(\varepsilon^{N+\alpha-\frac{(N-2)(p+q)}{2}}\right) \int_{B_{2 / \varepsilon}} \int_{B_{2 / \varepsilon}} \frac{1}{\left(1+|x|^{2}\right)^{\frac{(N-2) p}{2}}|x-y|^{N-\alpha}\left(1+|y|^{2}\right)^{\frac{(N-2) q}{2}}} d x d y \\
& \geq O\left(\varepsilon^{\frac{N+\alpha-(N-2) p}{2}}\right) \int_{B_{\frac{1}{2}}}\left[\int_{B_{x, \frac{1}{2}}} \frac{1}{\left(1+|y|^{2}\right)^{\frac{(N-2) q}{2}}|x-y|^{N-\alpha}} d y\right] \frac{1}{\left(1+|x|^{2}\right)^{\frac{(N-2) p}{2}}} d x \\
& \geq O\left(\varepsilon^{\frac{N+\alpha-(N-2) p}{2}}\right) \int_{B_{\frac{1}{2}}}\left[\int_{B_{x, \frac{1}{2}}} \frac{1}{|x-y|^{N-\alpha}} d y\right] \frac{1}{\left(1+|x|^{2}\right)^{\frac{(N-2) p}{2}}} d x \\
& =O\left(\varepsilon^{\frac{N+\alpha-(N-2) p}{2}}\right) \int_{B_{\frac{1}{2}}} \frac{1}{|y|^{N-\alpha}} d y \cdot \int_{B_{\frac{1}{2}}} \frac{1}{\left(1+|x|^{2}\right)^{\frac{(N-2) p}{2}}} d x=O\left(\varepsilon^{\frac{N+\alpha-(N-2) p}{2}}\right) .
\end{aligned}
$$

By direct computation, we have

$$
\mathcal{H}_{2}=O\left(\varepsilon^{\frac{(N-2)(p+q)}{2}}\right) \int_{B_{1}} \int_{B_{2} \backslash B_{1}} \frac{1}{\left(\varepsilon^{2}+|x|^{2}\right)^{\frac{(N-2) p}{2}}|x-y|^{N-\alpha}\left(\varepsilon^{2}+|y|^{2}\right)^{\frac{(N-2) q}{2}}} d x d y .
$$

By the Hardy-Littlewood-Sobolev inequality with $\frac{1}{r_{2}}+\frac{1}{s_{2}}=1+\frac{\alpha}{N}$ and

$$
s_{2} \in\left(\frac{N}{N+\alpha}, \frac{N}{N+\alpha-(N-2) p}\right)
$$

we have

$$
\begin{aligned}
\mathcal{H}_{2} & \leq O\left(\varepsilon^{\frac{(N-2)(p+q)}{2}}\right)\left(\int_{B_{2} \backslash B_{1}} \frac{1}{\left(\varepsilon^{2}+|x|^{2}\right)^{\frac{(N-2) p r_{2}}{2}}} d x\right)^{\frac{1}{r_{2}}}\left(\int_{B_{1}} \frac{1}{\left(\varepsilon^{2}+|y|^{2}\right)^{\frac{(N+\alpha) s_{2}}{2}}} d y\right)^{\frac{1}{s_{2}}} \\
& \leq O\left(\varepsilon^{\frac{(N-2)(p+q)}{2}-(N+\alpha)+\frac{N}{s_{2}}}\right)\left(\int_{0}^{\frac{1}{\varepsilon}} \frac{z^{N-1}}{\left(1+|z|^{2}\right)^{\frac{(N+\alpha) s_{2}}{2}}} d z\right)^{\frac{1}{s_{2}}} \\
& \leq O\left(\varepsilon^{\frac{N}{s_{2}}-\frac{(N+\alpha)-(N-2) p}{2}}\right)\left(1+\int_{1}^{\frac{1}{\varepsilon}}|z|^{N-1-(N+\alpha) s_{2}} d z\right)^{\frac{1}{s}}=O\left(\varepsilon^{\frac{N}{s_{2}}-\frac{(N+\alpha)-(N-2) p}{2}}\right) .
\end{aligned}
$$


By direct computation, we get

$$
\mathcal{H}_{3}=O\left(\varepsilon^{\frac{(N-2)(p+q)}{2}}\right) \int_{B_{2} \backslash B_{1}} \int_{B_{1}} \frac{1}{\left(\varepsilon^{2}+|x|^{2}\right)^{\frac{(N-2) p}{2}}|x-y|^{N-\alpha}\left(\varepsilon^{2}+|y|^{2}\right)^{\frac{(N-2) q}{2}}} d x d y
$$

By the Hardy-Littlewood-Sobolev inequality with $\frac{1}{r_{3}}+\frac{1}{s_{3}}=1+\frac{\alpha}{N}$ and

$$
s_{3}>\frac{N}{(N-2) p}
$$

we have

$$
\begin{aligned}
\mathcal{H}_{3} & \leq O\left(\varepsilon^{\frac{(N-2)(p+q)}{2}}\right)\left(\int_{B_{1}} \frac{1}{\left(\varepsilon^{2}+|x|^{2}\right)^{\frac{(N-2) p s_{3}}{2}}} d x\right)^{\frac{1}{s_{3}}}\left(\int_{B_{2} \backslash B_{1}} \frac{1}{\left(\varepsilon^{2}+|y|^{2}\right)^{\frac{(N+\alpha) r_{3}}{2}}} d y\right)^{\frac{1}{r_{3}}} \\
& \leq O\left(\varepsilon^{\frac{(N-2)(p+q)}{2}-(N-2) p+\frac{N}{s_{3}}}\right)\left(\int_{0}^{\frac{1}{\varepsilon}} \frac{z^{N-1}}{\left(1+|z|^{2}\right)^{\frac{(N-2) p s_{3}}{2}}} d z\right)^{\frac{1}{s_{3}}} \\
& \leq O\left(\varepsilon^{\frac{N}{s_{3}+\frac{N+\alpha-(N-2) p}{2}}}\right)\left(1+\int_{1}^{\frac{1}{\varepsilon}}|z|^{N-1-(N-2) p s_{3}} d z\right)^{\frac{1}{s_{3}}} \\
& =O\left(\varepsilon^{\frac{N}{s_{3}+\frac{N+\alpha-(N-2) p}{2}}}\right) .
\end{aligned}
$$

We also get

$$
\begin{aligned}
\mathcal{H}_{4} & =O\left(\varepsilon^{\frac{N+\alpha+(N-2) p}{2}}\right) \int_{B_{2} \backslash B_{1}} \int_{B_{2} \backslash B_{1}} \frac{1}{\left(\varepsilon^{2}+|x|^{2}\right)^{\frac{(N-2) p}{2}}|x-y|^{N-\alpha}\left(\varepsilon^{2}+|y|^{2}\right)^{\frac{(N-2) q}{2}}} d x d y \\
& \leq O\left(\varepsilon^{\frac{N+\alpha+(N-2) p}{2}}\right) \int_{B_{2} \backslash B_{1}} \int_{B_{2} \backslash B_{1}} \frac{1}{|x|^{(N-2) p}|x-y|^{N-\alpha}|y|^{(N-2) q}} d x d y \\
& \leq O\left(\varepsilon^{\frac{N+\alpha+(N-2) p}{2}}\right)\left[\int_{B_{2} \backslash B_{1}}|x|^{-\frac{2 p N(N-2)}{N+\alpha}} d x\right]^{\frac{N+\alpha}{2 N}} \cdot\left[\int_{B_{2} \backslash B_{1}}|x|^{-2 N} d x\right]^{\frac{N+\alpha}{2 N}} \\
& \leq O\left(\varepsilon^{\frac{N+\alpha+(N-2) p}{2}}\right) .
\end{aligned}
$$

Combining (9), (10), (11), and (12), we have

$$
\mathcal{H} \geq O\left(\varepsilon^{\frac{N+\alpha-(N-2) p}{2}}\right)-O\left(\varepsilon^{\frac{N}{s_{2}}-\frac{(N+\alpha)-(N-2) p}{2}}\right)-O\left(\varepsilon^{\frac{N}{s_{3}+\frac{N+\alpha-(N-2) p}{2}}}\right)-O\left(\varepsilon^{\frac{N+\alpha+(N-2) p}{2}}\right) .
$$

Noting that $s_{2}<\frac{N}{N+\alpha-(N-2) p}$, we obtain

$$
\mathcal{H} \geq O\left(\varepsilon^{\frac{N+\alpha-(N-2) p}{2}}\right)
$$


Li and Wang Boundary Value Problems

(2021) 2021:102

Page 10 of 20

Case $\mathcal{M}$ : By the definition of $u_{\varepsilon}(x)$, we have

$$
\begin{aligned}
\mathcal{M}= & \int_{\mathbb{R}^{N}} \int_{\mathbb{R}^{N}} \frac{\hat{C}\left|u_{\varepsilon}(x)\right|^{q}\left|u_{\varepsilon}(y)\right|^{q}}{|x-y|^{N-\alpha}} d x d y=\int_{B_{2}} \int_{B_{2}} \frac{\hat{C}\left|u_{\varepsilon}(x)\right|^{q}\left|u_{\varepsilon}(y)\right|^{q}}{|x-y|^{N-\alpha}} d x d y \\
\geq & \int_{B_{1}} \int_{B_{1}} \frac{\hat{C}\left|u_{\varepsilon}(x)\right|^{q}\left|u_{\varepsilon}(y)\right|^{q}}{|x-y|^{N-\alpha}} d x d y=\int_{B_{1}} \int_{B_{1}} \frac{\hat{C}\left|U_{\varepsilon}(x)\right|^{q}\left|U_{\varepsilon}(y)\right|^{q}}{|x-y|^{N-\alpha}} d x d y \\
= & \int_{\mathbb{R}^{N}} \int_{\mathbb{R}^{N}} \frac{\left|U_{\varepsilon}(x)\right|^{q}\left|U_{\varepsilon}(y)\right|^{q}}{|x-y|^{N-\alpha}} d x d y-2 \int_{\mathbb{R}^{N} \backslash B_{1}} \int_{B_{1}} \frac{\hat{C}\left|U_{\varepsilon}(x)\right|^{q}\left|U_{\varepsilon}(y)\right|^{q}}{|x-y|^{N-\alpha}} d x d y \\
& -\int_{\mathbb{R}^{N} \backslash B_{1}} \int_{\mathbb{R}^{N} \backslash B_{1}} \frac{\hat{C}\left|U_{\varepsilon}(x)\right|^{q}\left|U_{\varepsilon}(y)\right|^{q}}{|x-y|^{N-\alpha}} d x d y \\
\geq & \int_{\mathbb{R}^{N}} \int_{\mathbb{R}^{N}} \frac{\hat{C}\left|U_{\varepsilon}(x)\right|^{q}\left|U_{\varepsilon}(y)\right|^{q}}{|x-y|^{N-\alpha}} d x d y-2 \int_{\mathbb{R}^{N} \backslash B_{1}} \int_{B_{1}} \frac{\hat{C}\left|U_{\varepsilon}(x)\right|^{q}\left|U_{\varepsilon}(y)\right|^{q}}{|x-y|^{N-\alpha}} d x d y \\
& -\int_{\mathbb{R}^{N} \backslash B_{1}} \int_{\mathbb{R}^{N} \backslash B_{1}} \frac{\hat{C}\left|U_{\varepsilon}(x)\right|^{q}\left|U_{\varepsilon}(y)\right|^{q}}{|x-y|^{N-\alpha}} d x d y \\
= & \mathcal{S}_{1}^{\frac{N+\alpha}{2+\alpha}}-2 \mathcal{M}_{1}-\mathcal{M}_{2},
\end{aligned}
$$

where

$$
\mathcal{M}_{1}:=\int_{\mathbb{R}^{N} \backslash B_{1}} \int_{B_{1}} \frac{\hat{C}\left|U_{\varepsilon}(x)\right|^{q}\left|U_{\varepsilon}(y)\right|^{q}}{|x-y|^{N-\alpha}} d x d y
$$

and

$$
\mathcal{M}_{2}:=\int_{\mathbb{R}^{N} \backslash B_{1}} \int_{\mathbb{R}^{N} \backslash B_{1}} \frac{\hat{C}\left|U_{\varepsilon}(x)\right|^{q}\left|U_{\varepsilon}(y)\right|^{q}}{|x-y|^{N-\alpha}} d x d y .
$$

By direct computation, we have, for $\varepsilon<1$,

$$
\mathcal{M}_{1}=O\left(\varepsilon^{(N-2) q}\right) \int_{\mathbb{R}^{N} \backslash B_{1}} \int_{B_{1}} \frac{1}{\left(\varepsilon^{2}+|x|^{2}\right)^{\frac{(N-2) q}{2}}|x-y|^{N-\alpha}\left(\varepsilon^{2}+|y|^{2}\right)^{\frac{(N-2) q}{2}}} d x d y .
$$

By the Hardy-Littlewood-Sobolev inequality with $t=r=\frac{2 N}{N+\alpha}$ and $q=\frac{N+\alpha}{N-2}$, we have

$$
\begin{aligned}
\mathcal{M}_{1} & \leq O\left(\varepsilon^{(N-2) q}\right)\left(\int_{\mathbb{R}^{N} \backslash B_{1}} \frac{1}{\left(\varepsilon^{2}+|x|^{2}\right)^{N}} d x\right)^{\frac{N+\alpha}{2 N}}\left(\int_{B_{1}} \frac{1}{\left(\varepsilon^{2}+|y|^{2}\right)^{N}} d y\right)^{\frac{N+\alpha}{2 N}} \\
& \leq O\left(\varepsilon^{\frac{N+\alpha}{2}}\right)\left(\int_{1}^{+\infty} z^{-1-N} d z\right)^{\frac{N+\alpha}{2 N}}\left(\int_{0}^{\frac{1}{\varepsilon}} \frac{z^{N-1}}{\left(1+|z|^{2}\right)^{N}} d z\right)^{\frac{N+\alpha}{2 N}} \\
& \leq O\left(\varepsilon^{\frac{N+\alpha}{2}}\right) \cdot\left(1+\int_{1}^{\frac{1}{\varepsilon}} z^{-1-N} d z\right)^{\frac{N+\alpha}{2 N}} \\
& \leq O\left(\varepsilon^{\frac{N+\alpha}{2}}\right)\left(1+\left.\frac{1}{-N} r^{-N}\right|_{1} ^{\frac{1}{\varepsilon}}\right)^{\frac{N+\alpha}{2 N}} \leq O\left(\varepsilon^{\frac{N+\alpha}{2}}\right) .
\end{aligned}
$$


We also get

$$
\begin{aligned}
\mathcal{M}_{2} & =O\left(\varepsilon^{(N-2) q}\right) \int_{\mathbb{R}^{N} \backslash B_{1}} \int_{\mathbb{R}^{N} \backslash B_{1}} \frac{1}{\left(\varepsilon^{2}+|x|^{2}\right)^{\frac{(N-2) q}{2}}|x-y|^{N-\alpha}\left(\varepsilon^{2}+|y|^{2}\right)^{\frac{(N-2) q}{2}}} d x d y \\
& \leq O\left(\varepsilon^{(N-2) q}\right) \int_{\mathbb{R}^{N} \backslash B_{1}} \int_{\mathbb{R}^{N} \backslash B_{1}} \frac{1}{|x|^{(N-2) q}|x-y|^{N-\alpha}|y|^{(N-2) q}} d x d y \\
& \leq O\left(\varepsilon^{(N-2) q}\right)\left[\int_{\mathbb{R}^{N} \backslash B_{1}}|x|^{-2 N} d x\right]^{\frac{N+\alpha}{N}}=O\left(\varepsilon^{N+\alpha}\right) .
\end{aligned}
$$

Combining (14) and (15), we have

$$
\mathcal{M} \geq \mathcal{S}_{1}^{\frac{N+\alpha}{2+\alpha}}-O\left(\varepsilon^{\frac{N+\alpha}{2}}\right) .
$$

From (2), (3), (8), (13), and (16), we have

$$
\begin{aligned}
\Phi\left(t u_{\varepsilon}\right) \leq & \frac{t^{2}}{2}\left(\mathcal{S}_{1}^{\frac{N+\alpha}{2+\alpha}}+O\left(\varepsilon^{N-2}\right)\right)-\frac{t^{2 q}}{2 q^{2}}\left(\mathcal{S}_{1}^{\frac{N+\alpha}{2+\alpha}}-O\left(\varepsilon^{\frac{N+\alpha}{2}}\right)\right) \\
& +t^{2} h(\varepsilon)-t^{2 p}\left[O\left(\varepsilon^{N+\alpha-(N-2) p}\right)-O\left(\varepsilon^{\frac{N}{s_{1}}}\right)\right]-O\left(\varepsilon^{\frac{N+\alpha-(N-2) p}{2}}\right) t^{p+q} .
\end{aligned}
$$

It is easy to see that there exist constants $\bar{\varepsilon}>0$ and $t_{2}>t_{1}>0$ such that, for all $\varepsilon \in(0, \bar{\varepsilon})$ and $t \in\left[0, t_{1}\right] \cup\left[t_{2}, \infty\right)$,

$$
\Phi\left(t u_{\varepsilon}\right)<\frac{1}{2}\left(1-\frac{1}{q}\right) q^{\frac{1}{q-1}} \mathcal{S}_{1}^{\frac{N+\alpha}{2+\alpha}}
$$

In the following we may set $t \in\left[t_{1}, t_{2}\right]$ and $\varepsilon \in(0, \bar{\varepsilon})$. Then we have

$$
\begin{aligned}
\Phi\left(t u_{\varepsilon}\right) \leq & \frac{t^{2}}{2} \mathcal{S}_{1}^{\frac{N+\alpha}{2+\alpha}}-\frac{t^{2 q}}{2 q^{2}} \mathcal{S}_{1}^{\frac{N+\alpha}{2+\alpha}}+\frac{t^{2}}{2} O\left(\varepsilon^{N-2}\right)+\frac{t^{2 q}}{2 q^{2}} O\left(\varepsilon^{\frac{N+\alpha}{2}}\right) \\
& +t^{2} h(\varepsilon)-t^{2 p} O\left(\varepsilon^{N+\alpha-(N-2) p}\right)+t^{2 p} O\left(\varepsilon^{\frac{N}{S_{1}}}\right)-t^{p+q} O\left(\varepsilon^{\frac{N+\alpha-(N-2) p}{2}}\right) \\
\leq & \frac{t^{2}}{2} \mathcal{S}_{1}^{\frac{N+\alpha}{2+\alpha}}-\frac{t^{2 q}}{2 q^{2}} \mathcal{S}_{1}^{\frac{N+\alpha}{2+\alpha}}+\frac{t_{2}^{2}}{2} O\left(\varepsilon^{N-2}\right)+\frac{t_{2}^{2 q}}{2 q^{2}} O\left(\varepsilon^{\frac{N+\alpha}{2}}\right) \\
& +t_{2}^{2} h(\varepsilon)-t_{1}^{2 p} O\left(\varepsilon^{N+\alpha-(N-2) p}\right)+t_{2}^{2 p} O\left(\varepsilon^{\frac{N}{S_{1}}}\right)-t_{1}^{p+q} O\left(\varepsilon^{\frac{N+\alpha-(N-2) p}{2}}\right) .
\end{aligned}
$$

By the definition of $h(\varepsilon)$ and (22), for $\varepsilon$ small enough, we obtain

$$
\Phi\left(t u_{\varepsilon}\right)<\frac{1}{2}\left(1-\frac{1}{q}\right) q^{\frac{1}{q-1}} \mathcal{S}_{1}^{\frac{N+\alpha}{2+\alpha}} \quad \text { for all } t \geq 0,
$$

provided one of the following conditions holds:

(1) $N \geq 4$ and $p \in\left[\frac{N+\alpha}{N}, \frac{N+\alpha}{N-2}\right)$;

(2) $N=3$ and $p \in\left(1+\frac{\alpha}{N-2}, \frac{N+\alpha}{N-2}\right)$.

The proof is finished.

The constant $\mathcal{S}_{2}$ is defined by

$$
\mathcal{S}_{2}:=\inf \left\{\frac{|u|_{2}^{2}}{\left[\int_{\mathbb{R}^{N}}\left(I_{\alpha} *|u|^{p}\right)|u|^{p} d x\right]^{1 / p}}: u \in H^{1}\left(\mathbb{R}^{N}\right) \backslash\{0\}\right\}
$$


Li and Wang Boundary Value Problems

(2021) 2021:102

Page 12 of 20

and is attained by the functions

$$
v_{\sigma}(x)=\frac{\tilde{C} \sigma^{N / 2}}{\left(1+\sigma^{2}|x|^{2}\right)^{N / 2}}
$$

where $\sigma>0$ (see [6, Theorem 4.3]).

Lemma 2.3 Suppose that $N \geq 3$ and $p, q \in\left[\frac{N+\alpha}{N}, \frac{N+\alpha}{N-2}\right]$. There exists a positive constant $\sigma_{0}$ such that if $\sigma>\sigma_{0}$ then

$$
\Phi\left(t v_{\sigma}\right)<\frac{1}{2}\left(1-\frac{1}{p}\right) p^{\frac{1}{p-1}} \mathcal{S}_{2}^{\frac{p}{p-1}} \quad \text { for all } t \geq 0
$$

under $p=\frac{N+\alpha}{N}$ and one of the following conditions:

(1) $N>4+\alpha$ and $q \in\left(\frac{N+\alpha}{N}, \frac{N+\alpha}{N-2}\right]$,

(2) $N<4+\alpha$ and $q \in\left(\frac{N+\alpha}{N}, \frac{N+\alpha+4}{N}\right)$.

Proof According to the definition of $v_{\sigma}$, we have

$$
\begin{aligned}
\int_{\mathbb{R}^{N}}\left|v_{\sigma}(x)\right|^{2} d x & =\int_{\mathbb{R}^{N}} \frac{\tilde{C}^{2} \sigma^{N}}{\left(1+\sigma^{2}|x|^{2}\right)^{N}} d x \\
& =\int_{\mathbb{R}^{N}} \frac{\tilde{C}^{2}}{\left(1+|\sigma x|^{2}\right)^{N}} d(\sigma x)=\int_{\mathbb{R}^{N}} \frac{\tilde{C}^{2}}{\left(1+|x|^{2}\right)^{N}} d x=\mathcal{S}_{2}^{\frac{N+\alpha}{\alpha}}
\end{aligned}
$$

and for

$$
\begin{aligned}
& r \in\left[\frac{N+\alpha}{N}, \frac{N+\alpha}{N-2}\right], \\
& \int_{\mathbb{R}^{N}} \int_{\mathbb{R}^{N}} \frac{\hat{C}\left|v_{\sigma}(x)\right|^{r}\left|v_{\sigma}(y)\right|^{r}}{|x-y|^{N-\alpha}} d x d y \\
& \quad=\int_{\mathbb{R}^{N}} \int_{\mathbb{R}^{N}} \frac{\hat{C} \tilde{C}^{2 r} \sigma^{N r}}{\left(1+\sigma^{2}|x|^{2}\right)^{\frac{N r}{2}}|x-y|^{N-\alpha}\left(1+\sigma^{2}|y|^{2}\right)^{\frac{N r}{2}}} d x d y \\
& \quad=\sigma^{N r-(N+\alpha)} \int_{\mathbb{R}^{N}} \int_{\mathbb{R}^{N}} \frac{\hat{C} \tilde{C}^{2 r}}{\left(1+|x|^{2}\right)^{\frac{N r}{2}}|x-y|^{N-\alpha}\left(1+|y|^{2}\right)^{\frac{N r}{2}}} d x d y .
\end{aligned}
$$

Especially, for $r=p$, we have

$$
\int_{\mathbb{R}^{N}} \int_{\mathbb{R}^{N}} \frac{\hat{C}\left|v_{\sigma}(x)\right|^{p}\left|v_{\sigma}(y)\right|^{p}}{|x-y|^{N-\alpha}} d x d y=\mathcal{S}_{2}^{\frac{N+\alpha}{\alpha}}
$$

We also get

$$
\begin{aligned}
& \int_{\mathbb{R}^{N}}\left|\nabla v_{\sigma}\right|^{2} d x \\
& \quad=\int_{\mathbb{R}^{N}} \frac{\tilde{C}^{2} N^{2} \sigma^{N+4}|x|^{2}}{\left(1+\sigma^{2}|x|^{2}\right)^{N+2}} d x=\sigma^{2} \int_{\mathbb{R}^{N}} \frac{\tilde{C}^{2} N^{2}|x|^{2}}{\left(1+|x|^{2}\right)^{N+2}} d x
\end{aligned}
$$


and

$$
\begin{aligned}
& \int_{\mathbb{R}^{N}} \int_{\mathbb{R}^{N}} \frac{\left|v_{\sigma}(x)\right|^{p}\left|v_{\sigma}(y)\right|^{q}}{|x-y|^{N-\alpha}} d x d y \\
& =\int_{\mathbb{R}^{N}} \int_{\mathbb{R}^{N}} \frac{\hat{C} \tilde{C}^{p+q} \sigma^{\frac{N(p+q)}{2}}}{\left(1+\sigma^{2}|x|^{2}\right)^{\frac{N p}{2}}|x-y|^{N-\alpha}\left(1+\sigma^{2}|y|^{2}\right)^{\frac{N q}{2}}} d x d y \\
& =\sigma^{\frac{N q-(N+\alpha)}{2}} \int_{\mathbb{R}^{N}} \int_{\mathbb{R}^{N}} \frac{\hat{C} \tilde{C}^{p+q}}{\left(1+|x|^{2}\right)^{\frac{N p}{2}}|x-y|^{N-\alpha}\left(1+|y|^{2}\right)^{\frac{N q}{2}}} d x d y .
\end{aligned}
$$

By the definition of $\Phi$ and combining (17)-(21), we obtain

$$
\begin{aligned}
\Phi\left(t v_{\sigma}\right)= & \frac{t^{2}}{2} \int_{\mathbb{R}^{N}}\left[\left|\nabla v_{\sigma}\right|^{2}+v_{\sigma}^{2}\right] d x-\frac{1}{2} \int_{\mathbb{R}^{N}}\left(I_{\alpha} * F\left(t v_{\sigma}\right)\right) F\left(t v_{\sigma}\right) d x \\
= & \frac{t^{2}}{2} \int_{\mathbb{R}^{N}}\left|\nabla v_{\sigma}\right|^{2} d x+\frac{t^{2}}{2} \int_{\mathbb{R}^{N}} v_{\sigma}^{2} d x-\frac{t^{2 p}}{2 p^{2}} \int_{\mathbb{R}^{N}}\left(I_{\alpha} *\left|v_{\sigma}\right|^{p}\right)\left|v_{\sigma}\right|^{p} d x \\
& -\frac{t^{p+q}}{p q} \int_{\mathbb{R}^{N}}\left(I_{\alpha} *\left|v_{\sigma}\right|^{p}\right)\left|v_{\sigma}\right|^{q} d x-\frac{t^{2 q}}{2 q^{2}} \int_{\mathbb{R}^{N}}\left(I_{\alpha} *\left|v_{\sigma}\right|^{q}\right)\left|v_{\sigma}\right|^{q} d x \\
= & \left(\frac{t^{2}}{2}-\frac{t^{2 p}}{2 p^{2}}\right) \mathcal{S}_{2}^{\frac{N+\alpha}{\alpha}}+c_{1} \sigma^{2} t^{2}-c_{2} \sigma^{N q-(N+\alpha)} t^{2 q}-c_{3} \sigma^{\frac{N q-(N+\alpha)}{2}} t^{p+q},
\end{aligned}
$$

where $c_{1}, c_{2}$, and $c_{3}$ are positive constants. It is easy to see that there exist constants $\sigma_{1}>0$ and $t_{4}>t_{3}>0$ such that, for all $\sigma \in\left(0, \sigma_{1}\right)$ and $t \in\left[0, t_{3}\right] \cup\left[t_{4}, \infty\right)$,

$$
\Phi\left(t v_{\sigma}\right)<\frac{1}{2}\left(1-\frac{1}{p}\right) p^{\frac{1}{p-1}} \mathcal{S}_{2}^{\frac{N+\alpha}{\alpha}}
$$

In the following we set $t \in\left[t_{3}, t_{4}\right]$ and $\sigma \in\left(0, \sigma_{1}\right)$. Thus we have

$$
\Phi\left(t v_{\sigma}\right) \leq \frac{1}{2}\left(1-\frac{1}{p}\right) p^{\frac{1}{p-1}} \mathcal{S}_{2}^{\frac{N+\alpha}{\alpha}}+c_{1} \sigma^{2} t_{4}^{2}-c_{3} \sigma^{\frac{N q-(N+\alpha)}{2}} t_{3}^{p+q}
$$

By $q<\frac{N+\alpha+4}{N}$, there exists a positive constant $\sigma_{0}$ such that if $\sigma \in\left(0, \sigma_{0}\right)$ then

$$
\Phi\left(t v_{\sigma}\right)<\frac{1}{2}\left(1-\frac{1}{p}\right) p^{\frac{1}{p-1}} \mathcal{S}_{2}^{\frac{N+\alpha}{\alpha}} \quad \text { for all } t \geq 0
$$

Noting that if $N>\alpha+4$ then $\frac{N+\alpha}{N-2}<\frac{N+\alpha+4}{N}$; if $N<\alpha+4$ then $\frac{N+\alpha}{N-2}>\frac{N+\alpha+4}{N}$, the conclusion follows.

\section{Proof of the main theorem}

It is easy to prove that there exist $\beta, \rho>0$ and $v \in H_{r}^{1}\left(\mathbb{R}^{N}\right)$ such that

(i) $\inf _{\|u\|=\rho} \Phi(u)>\beta$;

(ii) $\|v\|>\rho$ and $\Phi(v)<0$.

Thus $\Phi$ has mountain pass geometry. Define the mountain pass level $c$ by

$$
c:=\inf _{\gamma \in \Gamma} \max _{0 \leq t \leq 1} \Phi(\gamma(t))
$$


where

$$
\Gamma=\left\{\gamma \in C\left([0,1], H_{r}^{1}\left(\mathbb{R}^{N}\right)\right): \gamma(0)=0, \gamma(1)=\nu\right\} .
$$

Combining Lemmas 2.2 and 2.3, we have the following conclusions:

(i) If $q=\frac{N+\alpha}{N-2}, N \geq 4$, and $p \in\left(\frac{N+\alpha}{N}, \frac{N+\alpha}{N-2}\right)$ or $N=3$ and $p \in\left(1+\frac{\alpha}{N-2}, \frac{N+\alpha}{N-2}\right)$, then

$$
c \in\left(0, \frac{1}{2}\left(1-\frac{1}{q}\right) q^{\frac{1}{q-1}} \mathcal{S}_{1}^{\frac{N+\alpha}{2+\alpha}}\right) .
$$

(ii) If $p=\frac{N+\alpha}{N}, N>4+\alpha$, and $q \in\left(\frac{N+\alpha}{N}, \frac{N+\alpha}{N-2}\right)$ or $N<4+\alpha$ and $q \in\left(\frac{N+\alpha}{N}, \frac{N+\alpha+4}{N}\right)$, then

$$
c \in\left(0, \frac{1}{2}\left(1-\frac{1}{p}\right) p^{\frac{1}{p-1}} \mathcal{S}_{2}^{\frac{N+\alpha}{\alpha}}\right) .
$$

(iii) If $q=\frac{N+\alpha}{N-2}, p=\frac{N+\alpha}{N}$, and $N>4+\alpha$, then

$$
c \in\left(0, \min \left\{\frac{1}{2}\left(1-\frac{1}{q}\right) q^{\frac{1}{q-1}} \mathcal{S}_{1}^{\frac{N+\alpha}{2+\alpha}}, \frac{1}{2}\left(1-\frac{1}{p}\right) p^{\frac{1}{p-1}} \mathcal{S}_{2}^{\frac{N+\alpha}{\alpha}}\right\}\right) .
$$

From Proposition 2.1 in [8], there exists a Pohozǎev-Palais-Smale sequence $\left\{u_{n}\right\}_{n \in \mathbb{N}}$ in $H_{r}^{1}\left(\mathbb{R}^{N}\right)$ such that, as $n \rightarrow \infty$,

$$
\left\{\begin{array}{l}
\Phi\left(u_{n}\right) \rightarrow c, \\
\Phi^{\prime}\left(u_{n}\right) \rightarrow 0 \quad \text { strongly in }\left(H_{r}^{1}\left(\mathbb{R}^{N}\right)\right)^{\prime}, \\
\mathcal{P}\left(u_{n}\right) \rightarrow 0 .
\end{array}\right.
$$

For every $n \in \mathbb{N}$,

$$
\Phi\left(u_{n}\right)-\frac{1}{N+\alpha} \mathcal{P}\left(u_{n}\right)=\frac{2+\alpha}{2(N+\alpha)} \int_{\mathbb{R}^{N}}\left|\nabla u_{n}\right|^{2} d x+\frac{\alpha}{2(N+\alpha)} \int_{\mathbb{R}^{N}}\left|u_{n}\right|^{2} d x .
$$

As the left-hand side is bounded, the sequence $\left\{u_{n}\right\}_{n \in \mathbb{N}}$ is bounded in $H_{r}^{1}\left(\mathbb{R}^{N}\right)$.

By extracting if necessary to a subsequence, we may assume that $u_{n} \rightarrow u$ in $H_{r}^{1}\left(\mathbb{R}^{N}\right)$. It is obvious that $u$ is a solution of problem (P). Thus

$$
\Phi(u)=\Phi(u)-\frac{1}{N+\alpha} \mathcal{P}(u)=\frac{2+\alpha}{2(N+\alpha)} \int_{\mathbb{R}^{N}}|\nabla u|^{2} d x+\frac{\alpha}{2(N+\alpha)} \int_{\mathbb{R}^{N}}|u|^{2} d x \geq 0 .
$$

Let $v_{n}=u_{n}-u$. By the Brezis-Lieb lemma (see [16, Lemma 1.32]),

$$
\int_{\mathbb{R}^{N}}\left|\nabla u_{n}\right|^{2} d x=\int_{\mathbb{R}^{N}}\left|\nabla v_{n}\right|^{2} d x+\int_{\mathbb{R}^{N}}|\nabla u|^{2} d x+o(1)
$$

and

$$
\int_{\mathbb{R}^{N}}\left|u_{n}\right|^{2} d x=\int_{\mathbb{R}^{N}}\left|v_{n}\right|^{2} d x+\int_{\mathbb{R}^{N}}|u|^{2} d x+o(1) .
$$

According to the situation of $p, q, \alpha$, and $N$, we divide the discussion into three cases. 
Case (i): $q=\frac{N+\alpha}{N-2}, N \geq 4$, and $p \in\left(\frac{N+\alpha}{N}, \frac{N+\alpha}{N-2}\right)$ or $N=3$ and $p \in\left(1+\frac{\alpha}{N-2}, \frac{N+\alpha}{N-2}\right)$.

From Lemma 2.1, Propositions 2.4 and 2.5 in [14], we see that

$$
\begin{aligned}
& \int_{\mathbb{R}^{N}}\left(I_{\alpha} *\left|u_{n}\right|^{p}\right)\left|u_{n}\right|^{p} d x=\int_{\mathbb{R}^{N}}\left(I_{\alpha} *|u|^{p}\right)|u|^{p} d x+o(1), \\
& \int_{\mathbb{R}^{N}}\left(I_{\alpha} *\left|u_{n}\right|^{q}\right)\left|u_{n}\right|^{q} d x=\int_{\mathbb{R}^{N}}\left(I_{\alpha} *\left|v_{n}\right|^{q}\right)\left|v_{n}\right|^{q} d x+\int_{\mathbb{R}^{N}}\left(I_{\alpha} *|u|^{q}\right)|u|^{q} d x+o(1),
\end{aligned}
$$

and

$$
\int_{\mathbb{R}^{N}}\left(I_{\alpha} *\left|u_{n}\right|^{p}\right)\left|u_{n}\right|^{q} d x=\int_{\mathbb{R}^{N}}\left(I_{\alpha} *|u|^{p}\right)|u|^{q} d x+o(1) .
$$

Then we have

$$
\begin{aligned}
\left\langle\Phi^{\prime}\left(u_{n}\right), u_{n}\right\rangle & =\int_{\mathbb{R}^{N}}\left[\left|\nabla u_{n}\right|^{2}+u_{n}^{2}\right] d x-\int_{\mathbb{R}^{N}}\left(I_{\alpha} * F\left(u_{n}\right)\right) f\left(u_{u}\right) d x \\
& =\left\langle\Phi^{\prime}(v), v\right\rangle+\int_{\mathbb{R}^{N}}\left[\left|\nabla v_{n}\right|^{2}+v_{n}^{2}\right] d x-\frac{1}{q} \int_{\mathbb{R}^{N}}\left(I_{\alpha} *\left|v_{n}\right|^{q}\right)\left|v_{n}\right|^{q} d x+o(1) .
\end{aligned}
$$

From $\left\langle\Phi^{\prime}(v), v\right\rangle=0$ and $\left\langle\Phi^{\prime}\left(u_{n}\right), u_{n}\right\rangle \rightarrow 0$

$$
\int_{\mathbb{R}^{N}}\left[\left|\nabla v_{n}\right|^{2}+v_{n}^{2}\right] d x=\frac{1}{q} \int_{\mathbb{R}^{N}}\left(I_{\alpha} *\left|v_{n}\right|^{q}\right)\left|v_{n}\right|^{q} d x+o(1) .
$$

We also have

$$
\begin{aligned}
\mathcal{P}\left(u_{n}\right)= & \frac{N-2}{2} \int_{\mathbb{R}^{N}}\left|\nabla u_{n}\right|^{2} d x+\frac{N}{2} \int_{\mathbb{R}^{N}} u_{n}^{2} d x-\frac{N+\alpha}{2} \int_{\mathbb{R}^{N}}\left(I_{\alpha} * F\left(u_{n}\right)\right) F\left(u_{n}\right) d x \\
= & \mathcal{P}(u)+\frac{N-2}{2} \int_{\mathbb{R}^{N}}\left|\nabla v_{n}\right|^{2} d x+\frac{N}{2} \int_{\mathbb{R}^{N}} v_{n}^{2} d x \\
& -\frac{N}{2 q} \int_{\mathbb{R}^{N}}\left(I_{\alpha} *\left|v_{n}\right|^{q}\right)\left|v_{n}\right|^{q} d x+o(1) .
\end{aligned}
$$

Thus

$$
\frac{N-2}{2} \int_{\mathbb{R}^{N}}\left|\nabla v_{n}\right|^{2} d x+\frac{N}{2} \int_{\mathbb{R}^{N}} v_{n}^{2} d x=\frac{N-2}{2 q} \int_{\mathbb{R}^{N}}\left(I_{\alpha} *\left|v_{n}\right|^{q}\right)\left|v_{n}\right|^{q} d x+o(1) .
$$

Combining (26) and (27), we deduce that

$$
\int_{\mathbb{R}^{N}}\left|\nabla v_{n}\right|^{2} d x=\frac{1}{q} \int_{\mathbb{R}^{N}}\left(I_{\alpha} *\left|v_{n}\right|^{q}\right)\left|v_{n}\right|^{q} d x+o(1)
$$

and

$$
\int_{\mathbb{R}^{N}} v_{n}^{2} d x=o(1)
$$

We may assume that

$$
\int_{\mathbb{R}^{N}}\left|\nabla v_{n}\right|^{2} d x \rightarrow a, \quad \frac{1}{q} \int_{\mathbb{R}^{N}}\left(I_{\alpha} *\left|v_{n}\right|^{q}\right)\left|v_{n}\right|^{q} d x \rightarrow a,
$$

where $a$ is a nonnegative constant. 
We claim that $a=0$. If $a \neq 0$, by the definition of $\mathcal{S}_{1}$, we have

$$
\int_{\mathbb{R}^{N}}\left|\nabla v_{n}\right|^{2} d x \geq \mathcal{S}_{1}\left[\int_{\mathbb{R}^{N}}\left(I_{\alpha} *\left|v_{n}\right|^{q}\right)\left|v_{n}\right|^{q} d x\right]^{1 / q} .
$$

It follows that $a \geq \mathcal{S}_{1}(q \cdot a)^{1 / q}$, which yields

$$
a \geq q^{\frac{1}{q-1}} \mathcal{S}_{1}^{\frac{N+\alpha}{2+\alpha}}
$$

Similarly to the discussion of (25), we have

$$
\begin{aligned}
\Phi\left(u_{n}\right) & =\frac{1}{2} \int_{\mathbb{R}^{N}}\left[\left|\nabla u_{n}\right|^{2}+u_{n}^{2}\right] d x-\frac{1}{2} \int_{\mathbb{R}^{N}}\left(I_{\alpha} * F\left(u_{n}\right)\right) F\left(u_{n}\right) d x \\
& =\Phi(u)+\frac{1}{2} \int_{\mathbb{R}^{N}}\left|\nabla v_{n}\right|^{2} d x-\frac{1}{2 q^{2}} \int_{\mathbb{R}^{N}}\left(I_{\alpha} *\left|v_{n}\right|^{q}\right)\left|v_{n}\right|^{q} d x+o(1) .
\end{aligned}
$$

It follows from $\Phi(u) \geq 0$ and (28) that

$$
\begin{aligned}
c & =\lim _{n \rightarrow \infty} \Phi\left(u_{n}\right) \\
& \geq \lim _{n \rightarrow \infty}\left[\frac{1}{2} \int_{\mathbb{R}^{N}}\left|\nabla v_{n}\right|^{2} d x-\frac{1}{2 q^{2}} \int_{\mathbb{R}^{N}}\left(I_{\alpha} *\left|v_{n}\right|^{q}\right)\left|v_{n}\right|^{q} d x\right] \\
& =\frac{1}{2}\left(1-\frac{1}{q}\right) q^{\frac{1}{q-1}} \mathcal{S}_{1}^{\frac{N+\alpha}{2+\alpha}},
\end{aligned}
$$

which contradicts (22). Hence $a=0$. This gives $v_{n} \rightarrow 0$ in $H_{r}^{1}\left(\mathbb{R}^{n}\right)$.

Case (ii): $p=\frac{N+\alpha}{N}, N>4+\alpha$, and $q \in\left(\frac{N+\alpha}{N}, \frac{N+\alpha}{N-2}\right)$ or $N<4+\alpha$ and $q \in\left(\frac{N+\alpha}{N}, \frac{N+\alpha+4}{N}\right)$.

From Lemma 2.1, Propositions 2.4 and 2.5 in [14], we see that

$$
\begin{aligned}
& \int_{\mathbb{R}^{N}}\left(I_{\alpha} *\left|u_{n}\right|^{p}\right)\left|u_{n}\right|^{p} d x=\int_{\mathbb{R}^{N}}\left(I_{\alpha} *\left|v_{n}\right|^{p}\right)\left|v_{n}\right|^{p} d x+\int_{\mathbb{R}^{N}}\left(I_{\alpha} *|u|^{p}\right)|u|^{p} d x+o(1), \\
& \int_{\mathbb{R}^{N}}\left(I_{\alpha} *\left|u_{n}\right|^{q}\right)\left|u_{n}\right|^{q} d x=\int_{\mathbb{R}^{N}}\left(I_{\alpha} *|u|^{q}\right)|u|^{q} d x+o(1),
\end{aligned}
$$

and

$$
\int_{\mathbb{R}^{N}}\left(I_{\alpha} *\left|u_{n}\right|^{p}\right)\left|u_{n}\right|^{q} d x=\int_{\mathbb{R}^{N}}\left(I_{\alpha} *|u|^{p}\right)|u|^{q} d x+o(1) .
$$

Then we have

$$
\begin{aligned}
\left\langle\Phi^{\prime}\left(u_{n}\right), u_{n}\right\rangle & =\int_{\mathbb{R}^{N}}\left[\left|\nabla u_{n}\right|^{2}+u_{n}^{2}\right] d x-\int_{\mathbb{R}^{N}}\left(I_{\alpha} * F\left(u_{n}\right)\right) f\left(u_{u}\right) d x \\
& =\left\langle\Phi^{\prime}(v), v\right\rangle+\int_{\mathbb{R}^{N}}\left[\left|\nabla v_{n}\right|^{2}+v_{n}^{2}\right] d x-\frac{1}{p} \int_{\mathbb{R}^{N}}\left(I_{\alpha} *\left|v_{n}\right|^{p}\right)\left|v_{n}\right|^{p} d x+o(1) .
\end{aligned}
$$

From $\left\langle\Phi^{\prime}(v), v\right\rangle=0$ and $\left\langle\Phi^{\prime}\left(u_{n}\right), u_{n}\right\rangle \rightarrow 0$,

$$
\int_{\mathbb{R}^{N}}\left[\left|\nabla v_{n}\right|^{2}+v_{n}^{2}\right] d x=\frac{1}{p} \int_{\mathbb{R}^{N}}\left(I_{\alpha} *\left|v_{n}\right|^{p}\right)\left|v_{n}\right|^{p} d x+o(1) .
$$


We also have

$$
\begin{aligned}
\mathcal{P}\left(u_{n}\right)= & \frac{N-2}{2} \int_{\mathbb{R}^{N}}\left|\nabla u_{n}\right|^{2} d x+\frac{N}{2} \int_{\mathbb{R}^{N}} u_{n}^{2} d x-\frac{N+\alpha}{2} \int_{\mathbb{R}^{N}}\left(I_{\alpha} * F\left(u_{n}\right)\right) F\left(u_{n}\right) d x \\
= & \mathcal{P}(u)+\frac{N-2}{2} \int_{\mathbb{R}^{N}}\left|\nabla v_{n}\right|^{2} d x+\frac{N}{2} \int_{\mathbb{R}^{N}} v_{n}^{2} d x \\
& -\frac{N-2}{2 p} \int_{\mathbb{R}^{N}}\left(I_{\alpha} *\left|v_{n}\right|^{p}\right)\left|v_{n}\right|^{p} d x+o(1) .
\end{aligned}
$$

Thus

$$
\frac{N-2}{2} \int_{\mathbb{R}^{N}}\left|\nabla v_{n}\right|^{2} d x+\frac{N}{2} \int_{\mathbb{R}^{N}} v_{n}^{2} d x=\frac{N}{2 p} \int_{\mathbb{R}^{N}}\left(I_{\alpha} *\left|v_{n}\right|^{p}\right)\left|v_{n}\right|^{p} d x+o(1) .
$$

Combining (30) and (31), we deduce that

$$
\int_{\mathbb{R}^{N}}\left|\nabla v_{n}\right|^{2} d x=o(1)
$$

and

$$
\int_{\mathbb{R}^{N}} v_{n}^{2} d x=\frac{1}{p} \int_{\mathbb{R}^{N}}\left(I_{\alpha} *\left|v_{n}\right|^{p}\right)\left|v_{n}\right|^{p} d x+o(1) .
$$

We may assume that

$$
\int_{\mathbb{R}^{N}} v_{n}^{2} d x \rightarrow b, \quad \frac{1}{p} \int_{\mathbb{R}^{N}}\left(I_{\alpha} *\left|v_{n}\right|^{p}\right)\left|v_{n}\right|^{p} d x \rightarrow b,
$$

where $b$ is a nonnegative constant.

We claim that $b=0$. If $b \neq 0$, by the definition of $\mathcal{S}_{2}$, we have

$$
\int_{\mathbb{R}^{N}}\left|v_{n}\right|^{2} d x \geq \mathcal{S}_{2}\left[\int_{\mathbb{R}^{N}}\left(I_{\alpha} *\left|v_{n}\right|^{p}\right)\left|v_{n}\right|^{p} d x\right]^{1 / p} .
$$

It follows that $b \geq \mathcal{S}_{2}(p \cdot b)^{1 / p}$, which yields

$$
b \geq p^{\frac{1}{p-1}} \mathcal{S}_{2}^{\frac{N+\alpha}{\alpha}} .
$$

Similarly to the discussion of (29), we have

$$
\begin{aligned}
\Phi\left(u_{n}\right) & =\frac{1}{2} \int_{\mathbb{R}^{N}}\left[\left|\nabla u_{n}\right|^{2}+u_{n}^{2}\right] d x-\frac{1}{2} \int_{\mathbb{R}^{N}}\left(I_{\alpha} * F\left(u_{n}\right)\right) F\left(u_{n}\right) d x \\
& =\Phi(u)+\frac{1}{2} \int_{\mathbb{R}^{N}} v_{n}^{2} d x-\frac{1}{2 p^{2}} \int_{\mathbb{R}^{N}}\left(I_{\alpha} *\left|v_{n}\right|^{p}\right)\left|v_{n}\right|^{p} d x+o(1) .
\end{aligned}
$$


It follows from $\Phi(u) \geq 0$, and (36) or (37) that

$$
\begin{aligned}
c & =\lim _{n \rightarrow \infty} \Phi\left(u_{n}\right) \\
\geq & \lim _{n \rightarrow \infty}\left[\frac{1}{2} \int_{\mathbb{R}^{N}} v_{n}^{2} d x-\frac{1}{2 p^{2}} \int_{\mathbb{R}^{N}}\left(I_{\alpha} *\left|v_{n}\right|^{p}\right)\left|v_{n}\right|^{p} d x\right] \\
& =\frac{1}{2}\left(1-\frac{1}{p}\right) p^{\frac{1}{p-1}} \mathcal{S}_{2}^{\frac{N+\alpha}{\alpha}}
\end{aligned}
$$

which contradicts (23). Hence $b=0$. This gives $v_{n} \rightarrow 0$ in $H_{r}^{1}\left(\mathbb{R}^{n}\right)$.

Case (iii): $q=\frac{N+\alpha}{N-2}, p=\frac{N+\alpha}{N}$, and $N>4+\alpha$.

From Lemma 2.1, Propositions 2.4 and 2.5 in [14], we see that

$$
\begin{aligned}
& \int_{\mathbb{R}^{N}}\left(I_{\alpha} *\left|u_{n}\right|^{p}\right)\left|u_{n}\right|^{p} d x=\int_{\mathbb{R}^{N}}\left(I_{\alpha} *\left|v_{n}\right|^{p}\right)\left|v_{n}\right|^{p} d x+\int_{\mathbb{R}^{N}}\left(I_{\alpha} *|u|^{p}\right)|u|^{p} d x+o(1), \\
& \int_{\mathbb{R}^{N}}\left(I_{\alpha} *\left|u_{n}\right|^{q}\right)\left|u_{n}\right|^{q} d x=\int_{\mathbb{R}^{N}}\left(I_{\alpha} *\left|v_{n}\right|^{q}\right)\left|v_{n}\right|^{q} d x+\int_{\mathbb{R}^{N}}\left(I_{\alpha} *|u|^{q}\right)|u|^{p} d x+o(1),
\end{aligned}
$$

and

$$
\int_{\mathbb{R}^{N}}\left(I_{\alpha} *\left|u_{n}\right|^{p}\right)\left|u_{n}\right|^{q} d x=\int_{\mathbb{R}^{N}}\left(I_{\alpha} *|u|^{p}\right)|u|^{q} d x+o(1) .
$$

Then we have

$$
\begin{aligned}
\left\langle\Phi^{\prime}\left(u_{n}\right), u_{n}\right\rangle= & \int_{\mathbb{R}^{N}}\left[\left|\nabla u_{n}\right|^{2}+u_{n}^{2}\right] d x-\int_{\mathbb{R}^{N}}\left(I_{\alpha} * F\left(u_{n}\right)\right) f\left(u_{u}\right) d x \\
= & \left\langle\Phi^{\prime}(v), v\right\rangle+\int_{\mathbb{R}^{N}}\left[\left|\nabla v_{n}\right|^{2}+v_{n}^{2}\right] d x-\frac{1}{p} \int_{\mathbb{R}^{N}}\left(I_{\alpha} *\left|v_{n}\right|^{p}\right)\left|v_{n}\right|^{p} d x \\
& -\frac{1}{q} \int_{\mathbb{R}^{N}}\left(I_{\alpha} *\left|v_{n}\right|^{q}\right)\left|v_{n}\right|^{q} d x+o(1) .
\end{aligned}
$$

From $\left\langle\Phi^{\prime}(v), v\right\rangle=0$ and $\left\langle\Phi^{\prime}\left(u_{n}\right), u_{n}\right\rangle \rightarrow 0$,

$$
\begin{aligned}
\int_{\mathbb{R}^{N}}\left[\left|\nabla v_{n}\right|^{2}+v_{n}^{2}\right] d x \\
=\frac{1}{p} \int_{\mathbb{R}^{N}}\left(I_{\alpha} *\left|v_{n}\right|^{p}\right)\left|v_{n}\right|^{p} d x+\frac{1}{q} \int_{\mathbb{R}^{N}}\left(I_{\alpha} *\left|v_{n}\right|^{q}\right)\left|v_{n}\right|^{q} d x+o(1) .
\end{aligned}
$$

We also have

$$
\begin{aligned}
\mathcal{P}\left(u_{n}\right)= & \frac{N-2}{2} \int_{\mathbb{R}^{N}}\left|\nabla u_{n}\right|^{2} d x+\frac{N}{2} \int_{\mathbb{R}^{N}} u_{n}^{2} d x-\frac{N+\alpha}{2} \int_{\mathbb{R}^{N}}\left(I_{\alpha} * F\left(u_{n}\right)\right) F\left(u_{n}\right) d x \\
= & \mathcal{P}(u)+\frac{N-2}{2} \int_{\mathbb{R}^{N}}\left|\nabla v_{n}\right|^{2} d x+\frac{N}{2} \int_{\mathbb{R}^{N}} v_{n}^{2} d x \\
& -\frac{N-2}{2 p} \int_{\mathbb{R}^{N}}\left(I_{\alpha} *\left|\nu_{n}\right|^{p}\right)\left|v_{n}\right|^{p} d x-\frac{N}{2 q} \int_{\mathbb{R}^{N}}\left(I_{\alpha} *\left|v_{n}\right|^{q}\right)\left|v_{n}\right|^{q} d x+o(1) .
\end{aligned}
$$


Thus

$$
\begin{aligned}
& \frac{N-2}{2} \int_{\mathbb{R}^{N}}\left|\nabla v_{n}\right|^{2} d x+\frac{N}{2} \int_{\mathbb{R}^{N}} v_{n}^{2} d x \\
& \quad=\frac{N}{2 p} \int_{\mathbb{R}^{N}}\left(I_{\alpha} *\left|v_{n}\right|^{p}\right)\left|v_{n}\right|^{p} d x+\frac{N-2}{2 q} \int_{\mathbb{R}^{N}}\left(I_{\alpha} *\left|v_{n}\right|^{q}\right)\left|v_{n}\right|^{q} d x+o(1) .
\end{aligned}
$$

Combining (34) and (35), we deduce that

$$
\begin{aligned}
& \int_{\mathbb{R}^{N}}\left|\nabla v_{n}\right|^{2} d x=\frac{1}{q} \int_{\mathbb{R}^{N}}\left(I_{\alpha} *\left|v_{n}\right|^{q}\right)\left|v_{n}\right|^{q} d x+o(1), \\
& \int_{\mathbb{R}^{N}} v_{n}^{2} d x=\frac{1}{p} \int_{\mathbb{R}^{N}}\left(I_{\alpha} *\left|v_{n}\right|^{p}\right)\left|v_{n}\right|^{p} d x+o(1) .
\end{aligned}
$$

We may assume that

$$
\begin{aligned}
& \int_{\mathbb{R}^{N}}\left|\nabla v_{n}\right|^{2} d x \rightarrow a, \quad \frac{1}{q} \int_{\mathbb{R}^{N}}\left(I_{\alpha} *\left|v_{n}\right|^{q}\right)\left|v_{n}\right|^{q} d x \rightarrow a, \\
& \int_{\mathbb{R}^{N}} v_{n}^{2} d x \rightarrow b, \quad \frac{1}{p} \int_{\mathbb{R}^{N}}\left(I_{\alpha} *\left|v_{n}\right|^{p}\right)\left|v_{n}\right|^{p} d x \rightarrow b,
\end{aligned}
$$

where $a, b$ are nonnegative constants.

We claim that $a=b=0$. We prove this by taking off any other cases: (1) $a \neq 0, b=0$; (2) $a=0, b \neq 0$; (3) $a \neq 0, b \neq 0$. If $a \neq 0$, by the definition of $\mathcal{S}_{1}$, we have

$$
\int_{\mathbb{R}^{N}}\left|\nabla v_{n}\right|^{2} d x \geq \mathcal{S}_{1}\left[\int_{\mathbb{R}^{N}}\left(I_{\alpha} *\left|v_{n}\right|^{q}\right)\left|v_{n}\right|^{q} d x\right]^{1 / q} .
$$

It follows that $a \geq \mathcal{S}_{1}(q \cdot a)^{1 / q}$, which yields

$$
a \geq q^{\frac{1}{q-1}} \mathcal{S}_{1}^{\frac{N+\alpha}{2+\alpha}}
$$

If $b \neq 0$, by the definition of $\mathcal{S}_{2}$, we have

$$
\int_{\mathbb{R}^{N}}\left|v_{n}\right|^{2} d x \geq \mathcal{S}_{2}\left[\int_{\mathbb{R}^{N}}\left(I_{\alpha} *\left|v_{n}\right|^{p}\right)\left|v_{n}\right|^{p} d x\right]^{1 / p} .
$$

It follows that $b \geq \mathcal{S}_{2}(p \cdot b)^{1 / p}$, which yields

$$
b \geq p^{\frac{1}{p-1}} \mathcal{S}_{2}^{\frac{N+\alpha}{\alpha}} .
$$

Similarly to the discussion of (33), we have

$$
\begin{aligned}
\Phi\left(u_{n}\right)= & \frac{1}{2} \int_{\mathbb{R}^{N}}\left[\left|\nabla u_{n}\right|^{2}+u_{n}^{2}\right] d x-\frac{1}{2} \int_{\mathbb{R}^{N}}\left(I_{\alpha} * F\left(u_{n}\right)\right) F\left(u_{n}\right) d x \\
= & \Phi(u)+\frac{1}{2} \int_{\mathbb{R}^{N}}\left|\nabla v_{n}\right|^{2} d x+\frac{1}{2} \int_{\mathbb{R}^{N}} v_{n}^{2} d x \\
& -\frac{1}{2 p^{2}} \int_{\mathbb{R}^{N}}\left(I_{\alpha} *\left|v_{n}\right|^{p}\right)\left|v_{n}\right|^{p} d x-\frac{1}{2 q^{2}} \int_{\mathbb{R}^{N}}\left(I_{\alpha} *\left|v_{n}\right|^{q}\right)\left|v_{n}\right|^{q} d x+o(1) .
\end{aligned}
$$


It follows from $\Phi(u) \geq 0$, and (36) or (37) that

$$
\begin{aligned}
c= & \lim _{n \rightarrow \infty} \Phi\left(u_{n}\right) \\
\geq & \lim _{n \rightarrow \infty}\left[\frac{1}{2} \int_{\mathbb{R}^{N}}\left|\nabla v_{n}\right|^{2} d x+\frac{1}{2} \int_{\mathbb{R}^{N}} v_{n}^{2} d x\right. \\
& \left.-\frac{1}{2 p^{2}} \int_{\mathbb{R}^{N}}\left(I_{\alpha} *\left|v_{n}\right|^{p}\right)\left|v_{n}\right|^{p} d x-\frac{1}{2 q^{2}} \int_{\mathbb{R}^{N}}\left(I_{\alpha} *\left|v_{n}\right|^{q}\right)\left|v_{n}\right|^{q} d x\right] \\
= & \frac{1}{2}\left(1-\frac{1}{q}\right) q^{\frac{1}{q-1}} \mathcal{S}_{1}^{\frac{N+\alpha}{2+\alpha}} \text { or } \frac{1}{2}\left(1-\frac{1}{p}\right) p^{\frac{1}{p-1}} \mathcal{S}_{2}^{\frac{N+\alpha}{\alpha}},
\end{aligned}
$$

which contradicts (24). Hence $a=b=0$. This gives $v_{n} \rightarrow 0$ in $H_{r}^{1}\left(\mathbb{R}^{n}\right)$.

Combining Cases (i)-(iii), we can assume, going if necessary to a subsequence, $u_{n} \rightarrow u$ in $H_{r}^{1}\left(\mathbb{R}^{N}\right)$. Clearly, $\Phi^{\prime}(u)=0$ and $\Phi(u)=c$. Thus problem (P) has a nontrivial critical point $u$.

Then, by the same approaches which appear in [8, Sect. 4], we obtain Theorem 1.1.

\section{Acknowledgements}

Not applicable.

Funding

Not applicable.

\section{Availability of data and materials}

Data sharing not applicable to this article as no data sets were generated or analysed during the current study.

\section{Declarations}

Competing interests

The authors declare that they have no competing interests.

Authors' contributions

All authors read and approved the final manuscript.

\section{Publisher's Note}

Springer Nature remains neutral with regard to jurisdictional claims in published maps and institutional affiliations.

Received: 1 July 2021 Accepted: 21 November 2021 Published online: 11 December 2021

\section{References}

1. Brezis, H., Nirenberg, L.: Positive solutions of nonlinear elliptic equations involving critical Sobolev exponents. Commun. Pure Appl. Math. 36, 437-477 (1983)

2. Gao, F., Yang, M.: On nonlocal Choquard equations with Hardy-Littlewood-Sobolev critical exponents. J. Math. Anal. Appl. 448, 1006-1041 (2017)

3. Jeanjean, L., Tanaka, K.: A remark on least energy solutions in $\mathbb{R}^{N}$. Proc. Am. Math. Soc. 131, 2399-2408 (2003)

4. Li, X., Ma, S.: Choquard equations with critical nonlinearities. Commun. Contemp. Math. 22(4), 1950023 (2020)

5. Li, X., Ma, S., Zhang, G.: Existence and qualitative properties of solutions for Choquard equations with a local term. Nonlinear Anal., Real World Appl. 45, 1-25 (2019)

6. Lieb, E., Loss, M.: Analysis, 2nd edn. Graduate Studies in Mathematics, vol. 14. Am. Math. Soc., Providence (2001)

7. Moroz, V., Van Schaftingen, J.: Groundstates of nonlinear Choquard equations: existence, qualitative properties and decay asymptotics. J. Funct. Anal. 265, 153-184 (2013)

8. Moroz, V., Van Schaftingen, J.: Existence of groundstates for a class of nonlinear Choquard equations. Trans. Am. Math. Soc. 367, 6557-6579 (2015)

9. Moroz, V., Van Schaftingen, J.: Groundstates of nonlinear Choquard equations: Hardy-Littlewood-Sobolev critical exponent. Commun. Contemp. Math. 17(5), 1550005 (2015)

10. Moroz, V., Van Schaftingen, J.: A guide to the Choquard equation. J. Fixed Point Theory Appl. 19(1), 773-813 (2017)

11. Palais, R.S.: The principle of symmetric criticality. Commun. Math. Phys. 69, 19-30 (1979)

12. Ruiz, D., Van Schaftingen, J.: Odd symmetry of least energy nodal solutions for the Choquard equation. J. Differ. Equ. $264,1231-1262(2018)$

13. Seok, J.: Nonlinear Choquard equations involving a critical local term. Appl. Math. Lett. 63, 77-87 (2017)

14. Seok, J.: Nonlinear Choquard equations: doubly critical case. Appl. Math. Lett. 76, 148-156 (2018)

15. Van Schaftingen, J., Xia, J.K.: Groundstates for a local nonlinear perturbation of the Choquard equations with lower critical exponent. J. Math. Anal. Appl. 464, 1184-1202 (2018)

16. Willem, M.: Minimax Theorems. Birkhäuser, Boston (1996) 